\title{
Modelling of Electrical Power Systems with Dynamic Phasors in Modelica
}

\author{
Tao Yang Serhiy Bozhko Greg Asher \\ University of Nottingham \\ University Park, Nottingham, NG7 2RD, UK \\ \{Tao.Yang, Serhiy.Bozhko, Greg.Asher\}@ nottingham.ac.uk
}

\begin{abstract}
The more-electric aircraft has been identified as the dominant trend for the future aircraft. With the development of power electronics, advanced control and drive systems, a great increase number of new electrical loads will be seen on-board. This paper presents an electrical power system (EPS) library developed in Modelica based on the dynamic phasor concept. The developed library uses a modular modelling concept. This makes the library more flexible and user-friendly. The application of developed library is also demonstrated through simulations of a MOET-architecture EPS.
\end{abstract}

Keywords: Dynamic Phasor; More-Electric Aircraft; Modelica

\section{Introduction}

The more-electric aircraft (MEA) has been identified as a dominant trend for the next-generation aircraft. The recent advance of power electronics, electrical drives and modern control theory makes it possible to replace many functions, which are conventionally managed by hydraulic, pneumatic and mechanical power, with electrical power driven devices [1]. This move offers reduced overall system weight, as well as increased efficiency, reliability and performance of the aircraft. The future EPS may take many forms: AC, DC, hybrid, frequency-wild, variable voltage, together with the possibility of novel connectivity topologies. To address the stability, availability and capability issues as well as to assess the performance of the power quality and transient behaviour, extensive simulation work is required to develop the EPS architectures.

Due to the switching behaviour of power electronic devices and its resulted higher harmonics, it is very time-consuming and even impractical to simulate a large-scale EPS with some non-linear and timevarying models. Considering the system dynamic frequency is normally much lower than that of higher harmonics, it is a common practice to neglect these higher harmonics when studying system dynamic behaviour. The average modelling technique, which removes the higher harmonics by averaging the variable during one fundamental period, has been widely used to model the EPS recently. In these average models, the variables are transformed from the three-phase $a b c$ frame to a synchronous rotating $d q$ frame (DQ0 models). The DQ0 model demonstrates good performance and high efficiency under balanced conditions [2]. This model, however, becomes very slow under unbalanced or faulty conditions. This is due to the second harmonic present in the DQ0 model under unbalanced conditions.

In this paper, a general averaging modelling technique, referred to as dynamic phasors (DP), is introduced and a model library based on this concept is developed in Modelica. The DPs are in nature some time-varying Fourier series coefficients. Compared with conventional phasors, the DP can model the system even it is not in the steady state. Truncating unimportant higher order harmonics and only considering the significant components, DP models are capable of retaining the dominant dynamic features of the EPS and suitable for transient performance studies. The slow variation of DPs allows for larger simulation time steps and results in faster simulations under both balanced and unbalanced conditions.

The software used to develop models is Dymola, standing for dynamic modelling laboratory. This software uses the open Modelica modelling language which allows users to freely create their own model libraries or modify the ready-made model libraries. This modelling language has been widely 
used in modelling electrical power systems [3, 4]. In contrast to data flow-oriented languages with directed inputs and outputs, such as the widely known Matlab/Simulink tool, Modelica employs an equation-based modelling technique and all the variables are treated equally. This avoids the algebraic loop issues frequently occurred in Matlab/Simulink. The equation-based modelling concept also results in a faster modelling process and a significantly increased re-usability, since the interconnection between models is easier and simpler than that of the signal-flow based modelling. There is no need to explicitly define the interface equations and predefine the input and output signals.

In this paper, the dynamic phasor concept is briefly introduced. The development of DP models in Modelica is explained using an RLC circuit. DP models of key EPS elements, such as generators, PWM converters etc. are introduced. The application of the DP library is demonstrated through simulation of the MOET architecture EPS.

\section{Dynamic Phasors}

Before introducing the developed library, a brief introduction of the dynamic phasor concept is introduced in this section. The DPs essentially are some time-varying Fourier coefficients. For a timedomain quasi-periodic waveform $\mathrm{x}(\tau)$, defining a time-moving window $\tau \in(\mathrm{t}-\mathrm{T}, \mathrm{t}]$, as shown in Figure 1 , and viewing the waveforms in this window to be periodic, the Fourier expansion of the waveform in this interval can be represented by the following Fourier series [5]:

$$
x(\tau)=\sum_{k=-\infty}^{\infty} X_{k}(t) e^{j k \omega_{s} \tau}
$$

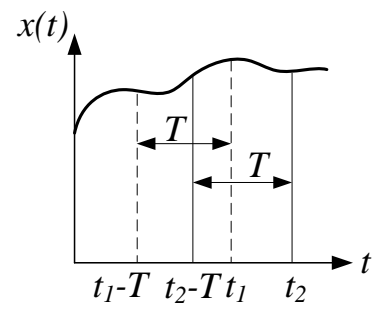

(a)

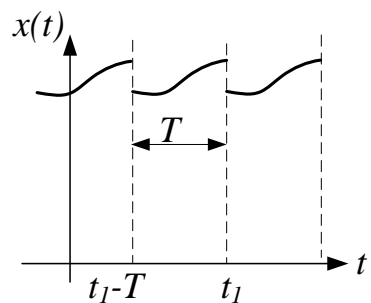

(b)
Figure 1 (a) Defined moving window at time $t_{1}$ and $t_{2}$, (b) equivalent periodic signal at time $t_{1}$ where $\omega_{s}=2 \pi / T$ and $T$ is the length of the window. Though the window length $\mathrm{T}$ can be an arbitrary value, it is common that the fundamental period of the signal is chosen to avoid the DPs spreading over the entire frequency axis. $X_{k}(\mathrm{t})$ is the kth Fourier coefficient in a complex form and is referred to as a "dynamic phasor". It is defined as follows:

$$
X_{k}(t)=\frac{1}{T} \int_{t-T}^{t} x(\tau) e^{-j k \omega_{s} \tau} d \tau=\langle x\rangle_{k}
$$

where $k$ can be any integer and is called the DP index. The triangular pair ' $\langle$ ' ' is used as the DP calculation symbols for any time-domain variables. In contrast to the traditional Fourier coefficients, these Fourier coefficients are time-varying as the integration interval (window) slides with time. The selected set of DPs, or $K$ with $k \in K$, defines the approximation accuracy of the waveform. For example, for DC-like variables and signals the index set only includes the component $k=0$, and for purely sinusoidal waveforms, with the window length equal to one period gives $k=1$.

A key factor in developing DP models is the relation between the derivatives of the variable $x(t)$ and the derivatives of kth Fourier coefficients given as:

$$
\left\langle\frac{d x}{d t}\right\rangle_{k}=\frac{d\langle x\rangle_{k}}{d t}+j k \omega_{s}\langle x\rangle_{k}
$$

It is important to notice that, the derivative term on the right side of (3) allows the DP to study the electromagnetic transients of the power system, not limited to steady-state studies. If we drop this derivative term and fix the DP index to be $k=1$, the DP will reduce to the traditional phasors, which is widely used to study steady-state or quasi-steady power systems.

\section{Modelica Library}

In this session, the DP library in Modelica will be introduced. An RLC circuit, which is used to represent the transmission line in many cases, has been chosen to illustrate the DP technique. DP models of other essential elements are detailed in our previous publications [6-10] and will be briefly reviewed in this session as well. 


\subsection{DP model of RLC circuits}

The DP models of RLC components are based on their time-domain voltage dynamic equations. Using the DP definition and properties, the DP transformation can be achieved conveniently.

\section{(i) Resistance element}

The time-domain voltage equation for a resistor can be expressed by:

$$
v=R i
$$

If the resistance $R$ is constant and time invariant, it can be moved out from the integration symbol and we obtain the DP form as:

$$
\langle v\rangle_{k}=R\langle i\rangle_{k}
$$

In our model, the DP index is chosen at $K=\{0,1\}$, the DP model in Modelica is shown below:

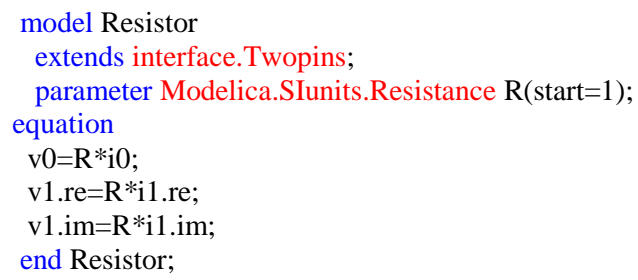

where $\mathrm{v} 0$ and $\mathrm{v} 1$ are corresponding to DPs $\langle v\rangle_{0,1}$.

\section{(ii) Inductance element}

The time-domain voltage equation for an inductor is written as:

$$
v=L \frac{d i}{d t}
$$

The DP form of (5) can be obtained by employing the DP definition and its differential as:

$$
\langle v\rangle_{k}=\left\langle L \frac{d i}{d t}\right\rangle_{k}=L \frac{d\langle i\rangle_{k}}{d t}+j \omega L\langle i\rangle_{k}
$$

In Modelica, the DP model for inductors is written as:

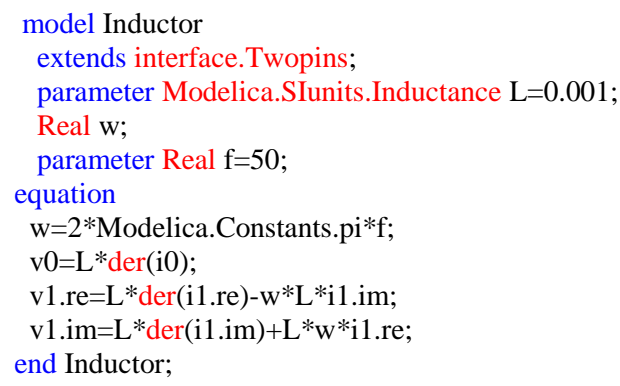

\section{(iii) Capacitance element}

The DP model for a capacitor can be derived the same way as that for the inductor and is written as:

$$
\langle i\rangle_{k}=C \frac{d\langle v\rangle_{k}}{d t}+j \omega C\langle v\rangle_{k}
$$

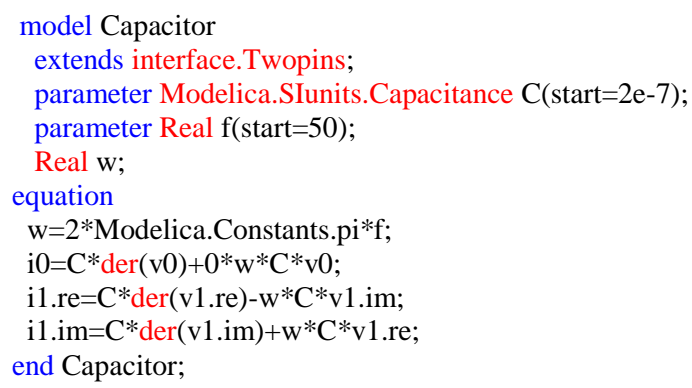

The application of the DP models in Modelica is very convenient. In reality, it even looks the same as the models in Modelica/Dymola standard libraries. A simple RLC circuit with all the elements modelled in DPs is shown in Figure 2.

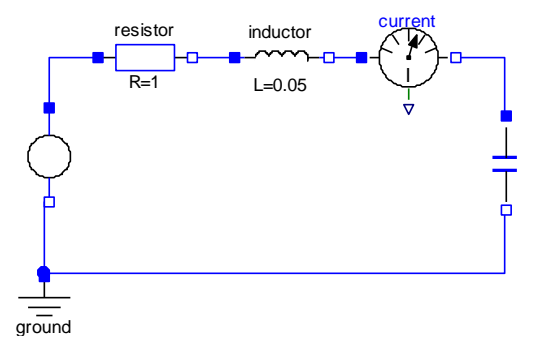

Figure 2 DP represented RLC circuit in Modelica
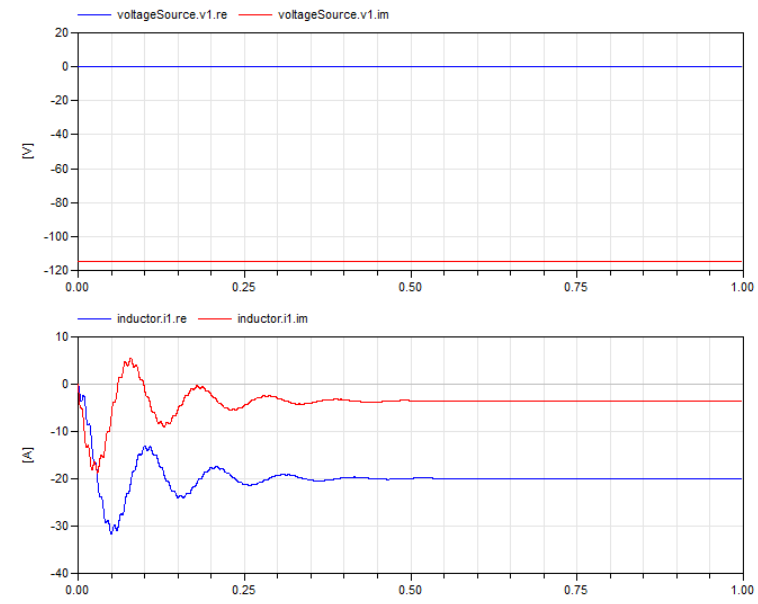

Figure 3 Simulation results of DP-modelled RLC circuit

The simulation results of the circuit are shown below. As can be seen, the sinusoidal source in DP domain becomes complex constant DC source. The currents flowing through the inductor turn into constant in the DP model. 


\subsection{DP models of synchronous generators and control}

The modelling of synchronous machines has been an important topic in power system engineering for many decades. Today there are a large number of different models used in different studies. The three-stage generator system in a conventional aircraft is simplified into a synchronous generator (SG). Figure 4 showed the SG with its controlling structure.

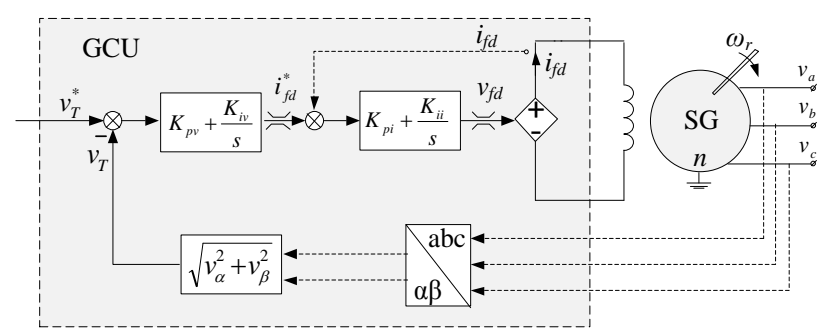

Figure 4 The equivalent circuit for the generating system in aircraft

The DP model of these machines is based on their time-domain voltage and flux equations. The fully detailed DP model of a synchronous machine has been can be found in [6] and will briefly introduced here. In DP domain, the synchronous machine can be modelled as:

$$
\begin{aligned}
\left\langle\mathbf{v}_{a b c s}\right\rangle_{k} & =-\mathbf{r}_{s}\left\langle\mathbf{i}_{a b c s}\right\rangle_{k}+\frac{d\left\langle\boldsymbol{\lambda}_{a b c s}\right\rangle_{k}}{d t}+j \omega\left\langle\boldsymbol{\lambda}_{a b c s}\right\rangle_{k} \\
\left\langle\mathbf{v}_{d q r}\right\rangle_{k} & =\mathbf{r}_{\mathbf{r}}\left\langle\mathbf{i}_{d q r}\right\rangle_{k}+\frac{d\left\langle\boldsymbol{\lambda}_{d q r}\right\rangle_{k}}{d t}+j \omega\left\langle\boldsymbol{\lambda}_{d q r}\right\rangle_{k} \\
\left\langle\boldsymbol{\lambda}_{a b c s}\right\rangle_{k} & =-\sum_{m}\left\langle\mathbf{L}_{s}\right\rangle_{m}\left\langle\mathbf{i}_{a b c s}\right\rangle_{k-m}+\sum_{n}\left\langle\mathbf{L}_{s r}\right\rangle_{n}\left\langle\mathbf{i}_{d q r}\right\rangle_{k-n} \\
\left\langle\boldsymbol{\lambda}_{d q r}\right\rangle_{k} & \left.=\sum_{m}\left\langle\mathbf{L}_{r s}\right\rangle_{m}\left\langle\mathbf{i}_{a b c s}\right\rangle_{k-m}+\sum_{n}\left\langle\mathbf{L}_{r}\right\rangle_{n} \mathbf{i}_{d q r}\right\rangle_{k-n}
\end{aligned}
$$

where $\mathbf{L}_{\mathrm{ss}}$ denotes the stator self-inductance matrix, $\mathbf{L}_{\mathrm{rr}}$ is the rotor self-inductance, $\mathbf{L}_{\mathrm{sr}}$ and $\mathbf{L}_{\mathrm{rs}}$ are the mutual inductance. The matrix $\mathbf{r}_{\mathrm{s}}$ is the resistance of stator windings and $\mathbf{r}_{\mathbf{r}}$ is the resistance of rotor windings.

The proportional-integral (PI) controllers can be converted into the DP domain with their statespace equations:

$$
\begin{gathered}
\dot{x}=k_{i} u \\
y=k_{p} u+x
\end{gathered}
$$

where $\mathrm{u}$ is the input, $\mathrm{x}$ is the state variable, and $k_{p}$ and $k_{i}$ are the proportional and integral gains correspondingly. This equation can be converted into dynamic phasors as:

$$
\begin{gathered}
\frac{d\langle x\rangle_{k}}{d t}=k_{i}\langle u\rangle_{k}-j k \omega\langle x\rangle_{k} \\
\langle y\rangle_{k}=k_{p}\langle u\rangle_{k}+\langle x\rangle_{k}
\end{gathered}
$$

The model of PI controller in modelica is written as:

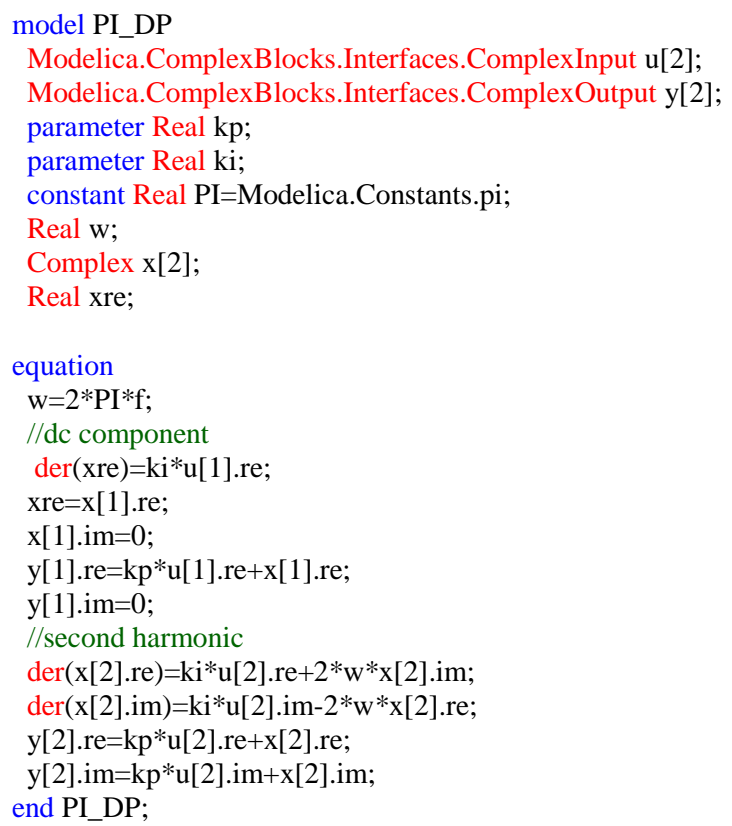

\subsection{Auto-transformer rectifier units}

The multi-pulse rectifier unit is widely used to supply the HVDC bus in aircraft electrical power systems. The DP modelling of an 18-pulse autotransformer rectifier unit (ATRU) has been discussed in our previous publication [11]. The symmetry of the ATRU is used to simplify the modelling process. The DP index for variables at the AC side terminals are set at $K=\{1\}$. For the DC terminal variables, the DP index is set at $K=\{0\}$ which is equal to their time-domain values. The DP model of ATRU is shown in Figure 5 and Figure 6. 


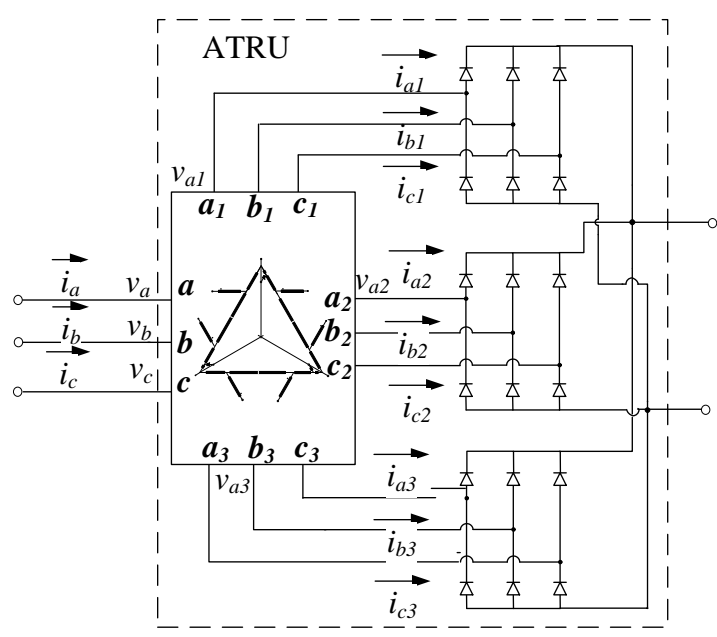

Figure 5 configuration of an 18-pulse ATRU

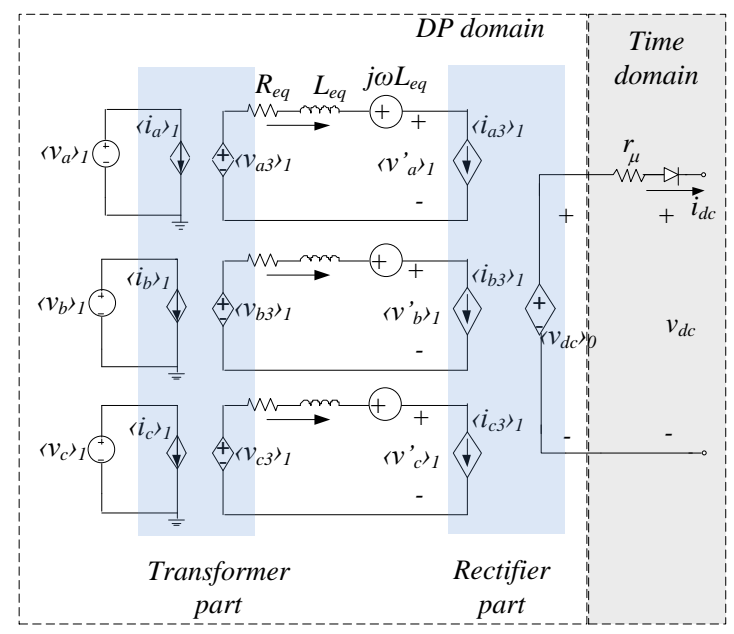

Figure 6 Equivalent circuit representation of DP model of an 18-pulse ATRU

\subsection{Controlled rectifier units}

The PWM controlled rectifier unit is well-known from previous publications and the topology is shown in Figure 7. With the voltage vector aligned with the $d$ axis in a synchronous rotating frame, denoted as the $D Q$ frame, the projections of the current vector onto the $D$ and $Q$ axes correspond to the active power and reactive power components respectively. This allows independent control of the active and reactive power flow. The electrical converter of the CRU is represented with the voltage and current relations in DP forms. The vector control of the CRU is transformed into DPs with the same strategy used in the controlled synchronous machines. The detailed development of the DP model for CRUs has been introduced in our previous publication [7].component is considered. Thus, the DP index is set at $K=\{0\}$.

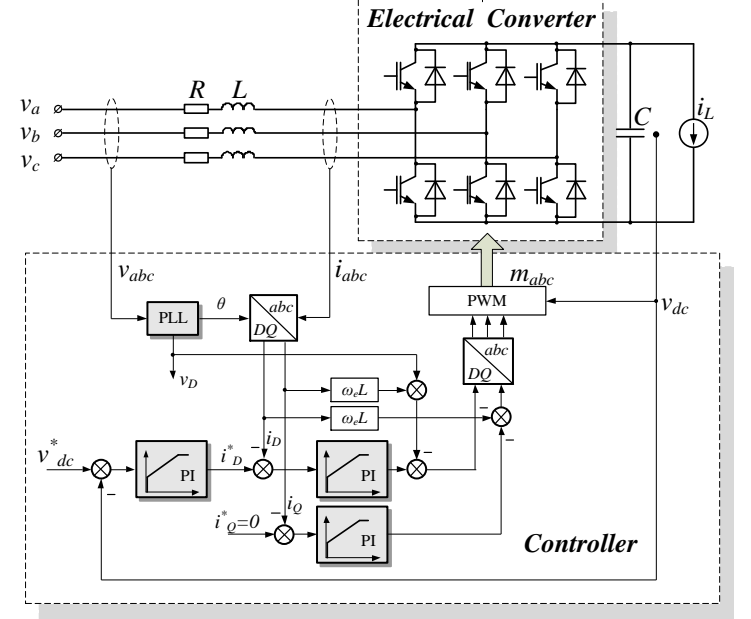

Figure 7 Structure of the PWM controlled rectifier

\section{System Simulation}

This simulation scheme has been shown in Figure 8. This EPS structure is based on the MOET large aircraft EPS architecture from Airbus France (document WP3.11 architecture V0 [12]). In the simulation studies, the electrical frequency of the SG1 is fixed at $400 \mathrm{~Hz}$ and $\mathrm{SG} 2$ fixed at $405 \mathrm{~Hz}$. The HVAC bus voltages are controlled at 230V RMS. The power rating of elements in the system is shown in Table 1.

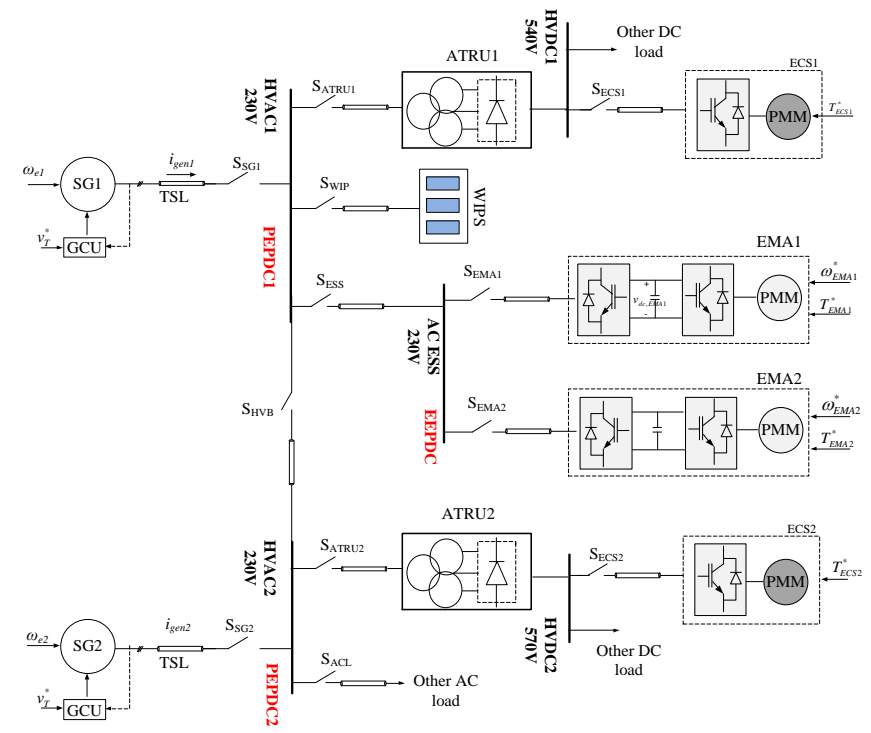

Figure 8 The EPS of the twin-generator aircraft 
Table 1 Rated power of equipment in the example EPS

\begin{tabular}{cc}
\hline \hline Equipment & Power rating \\
\hline ATRU & $150 \mathrm{~kW}$ \\
\hline WIPS & $60 \mathrm{~kW}$ \\
\hline ECS & $30 \mathrm{~kW}$ \\
\hline EMA & $5 \mathrm{~kW}$ \\
\hline AC essential bus fed CRU & $10 \mathrm{~kW}$ \\
\hline HVAC2 bus fed resistance & $9 \mathrm{~kW}$ \\
\hline HVDC bus fed resistance & $5 \mathrm{~kW}$ \\
\hline
\end{tabular}

Table 2 Simulation scenarios of twin-generator aircraft EPS under normal operation conditions

\begin{tabular}{cl}
\hline $\begin{array}{c}\text { Time } \\
(\mathrm{s})\end{array}$ & \multicolumn{1}{c}{ Events } \\
\hline 0.00 & Simulation starts; EPS starts up \\
\hline 0.15 & $\begin{array}{l}\text { Switches } \mathrm{S}_{\mathrm{SG} 1} \text { and } \mathrm{S}_{\mathrm{SG} 2} \text { are closed; Switch- } \\
\text { es } \mathrm{S}_{\mathrm{ATRU}} \text { and } \mathrm{S}_{\mathrm{ATRU}} \text { are closed; DC loads } \\
\text { on HVDC buses are connected }\end{array}$ \\
\hline 0.20 & $\begin{array}{l}\text { ESC1 and ECS2 start to accelerate to the } \\
\text { rated speed (3000rpm) }\end{array}$ \\
\hline 0.50 & $\begin{array}{l}\text { Rated load torques applied to ECS1 and } \\
\text { ECS2 (95Nm) }\end{array}$ \\
\hline 0.70 & WIPS changes from 60kW to 6kW \\
\hline 0.80 & $\begin{array}{l}\text { EMA1 and EMA2 start to accelerate to the } \\
\text { rated speed (900rpm) }\end{array}$ \\
\hline 0.90 & $\begin{array}{l}\text { Rated load torque applied to EMA1 and } \\
\text { EMA2 (54Nm) }\end{array}$ \\
\hline 1.00 & SG1 and SG2 are connected \\
\hline 1.02 & $\begin{array}{l}\mathrm{S}_{\mathrm{SG} 1} \text { opens; SG1 removes from the system } \\
\text { and SG2 covers the whole load system }\end{array}$ \\
\hline 1.20 & Simulation ends \\
\hline \hline
\end{tabular}

The simulation starts at $\mathrm{t}=0 \mathrm{~s}$. The GCU starts to regulate the HVAC bus phase voltage to $230 \mathrm{Vrms}$. After the HVAC bus voltage reaches the steady state, the switches $S_{A T R U 1}$ and $S_{A T R U 2}$ are closed at $\mathrm{t}=0.15 \mathrm{~s}$. The SG1 starts to supply ATRU1 through HVAC1 bus. At the same time, the SG2 starts to supply ATRU2 through HVAC2 bus. At $\mathrm{t}=0.2 \mathrm{~s}$, the speed reference for two ECS drive system is set at 3000rpm and the rated load torque $95 \mathrm{Nm}$ applied to these ECS systems at $\mathrm{t}=0.5 \mathrm{~s}$. At $\mathrm{t}=0.7 \mathrm{~s}$, the de-icing system starts to run at rated power and the WIPS is set at $60 \mathrm{~kW}$. After $50 \mathrm{~ms}$ second, the deicing process finishes and the power requirement of WIPS is reduced to $6 \mathrm{~kW}$ to maintain the temperature of the aircraft wings. The DC-link voltage reference of EMA is set to $800 \mathrm{~V}$ at $\mathrm{t}=0.6 \mathrm{~s}$. The speed reference of the EMA is set to rated speed $900 \mathrm{rpm}$ at $\mathrm{t}=0.8 \mathrm{~s}$ with rated load applied at $\mathrm{t}=0.9 \mathrm{~s}$. In order to demonstrate parallel operation of the two generators, $\mathrm{S}_{\mathrm{HVB}}$ is closed at $\mathrm{t}=1.0 \mathrm{~s}$. The two generators start to work in parallel for a short period, $20 \mathrm{~ms}$, then $\mathrm{S}_{\mathrm{HVB}}$ opens and the two generators work separately again. The event sequence of startup of the twin-generator aircraft EPS is also shown in Table 2.

Results from the ABC model (the model as a benchmark, in the three-phase coordination with switching behaviour) and DP models (all the elements are modelled in DPs) are compared in the following figures. The dynamic responses of ATRU-fed HVDC bus voltages, $v_{H V D C l}$ and $v_{H V D C 2}$ are shown in Figure 9. The initial values of $v_{H V D C l}$ and $v_{H V D C 2}$ are set at zero. At $\mathrm{t}=0.15 \mathrm{~s}$, when the switches $\mathrm{S}_{\mathrm{SG} 1}$ and $\mathrm{S}_{\mathrm{SG} 2}, S_{A T R U 1}, S_{A T R U 2}$ are closed, the inrush current push the DC-link voltage of ATRUs $v_{H V D C 1}$ and $v_{H V D C 2}$ from $0 \mathrm{~V}$ to around $800 \mathrm{~V}$. From $\mathrm{t}=0.2 \mathrm{~s}$, the ECS starts to speed up and draws power from the generator. A slope voltage drop at HVDC bus can be noticed from this point. This is because the PMSM in the ECS acquiring a linearly increasing power from the generator as shown in Figure 10 and Figure 11. The linearly increasing AC currents resulted in correspondingly a linearly voltage drop in the transmission lines and at AC terminals of ATRUs. This results in a linear decrease of the DC voltage $v_{H V D C l}$ and $v_{H V D C 2}$. When the rated loads of ECS1 and ECS2 are applied, a slight voltage drop can also be seen in $v_{H V D C 1}$ and $v_{H V D C 2}$ at $\mathrm{t}=0.5 \mathrm{~s}$. The reduction of the WIPS power requirement at $\mathrm{t}=0.7 \mathrm{~s}$ results in a higher $v_{H V D C l}$ and $v_{H V D C 2}$. When the generators SG1 and SG2 are connected, $v_{H V D C l}$ and $v_{H V D C 2}$ decrease due to the difference between $v_{H V A C I}$ and $v_{H V A C 2}$. With SG1 removed from the system at $\mathrm{t}=1.02 \mathrm{~s}$, SG2 starts to supply the whole load system and the system comes to the steady state after a short transient period. The $v_{H V D C l}$ and $v_{H V D C 2}$ from ABC and DP modelling techniques are well-matched as shown in Figure 9.

Since the system is assumed to be balanced, the currents flowing into ATRUs $i_{\text {ATRU1 }}$ and $i_{\text {ATRU2 }}$ are represented by the phase A current only. For comparison studies, the variables in the DP model are 
transformed to the time domain. The simulation results of $i_{\text {ATRU1 }}$ and $i_{\text {ATRU2 }}$ are shown in Figure 10 and Figure 11 . The currents $i_{\text {ATRU1 }}$ and $i_{\text {ATRU2 }}$ remains zero until the load connected to the HVDC buses at $\mathrm{t}=0.15 \mathrm{~s}$. The acceleration of PMSMs of ECS's induces the increasing of $i_{\text {ATRU1 }}$ and $i_{\text {ATRU2 }}$ starting from $\mathrm{t}=0.2 \mathrm{~s}$. The application of rated loads at ECS's introduces the step of $i_{\text {ATRU1 }}$ and $i_{\text {ATRU2 }}$. It can be seen that the results from $\mathrm{ABC}$ and $\mathrm{DP}$ models are well matched during the whole simulation process. The magnitude of DPs $\left\langle i_{A T R U 1}\right\rangle_{1}$ and $\left\langle i_{\text {ATRU2 }}\right\rangle_{1}$ are also shown in Figure 10 and Figure 11. From these two figures, it can be seen that the magnitudes of $\left\langle i_{\text {ATRU1 }}\right\rangle_{1}$ and $\left\langle i_{\text {ATRU2 }}\right\rangle_{1}$ give the envelope of the result from the ABC model.
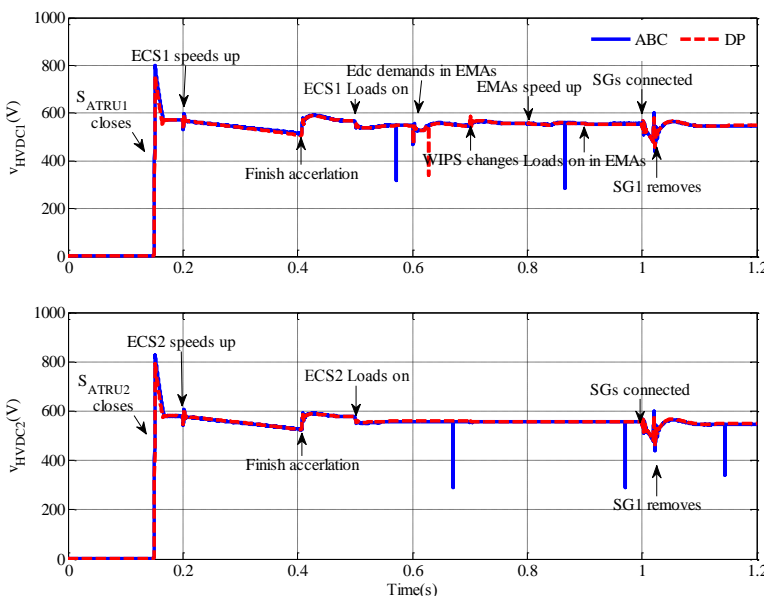

Figure 9 The dynamic response of $\mathrm{v}_{\mathrm{HVDC} 1}$ and $\mathrm{v}_{\mathrm{HVDC}}$. Above: response of $\mathrm{v}_{\mathrm{HVDC}}$; below: response of $\mathrm{V}_{\mathrm{HVDC} 2}$

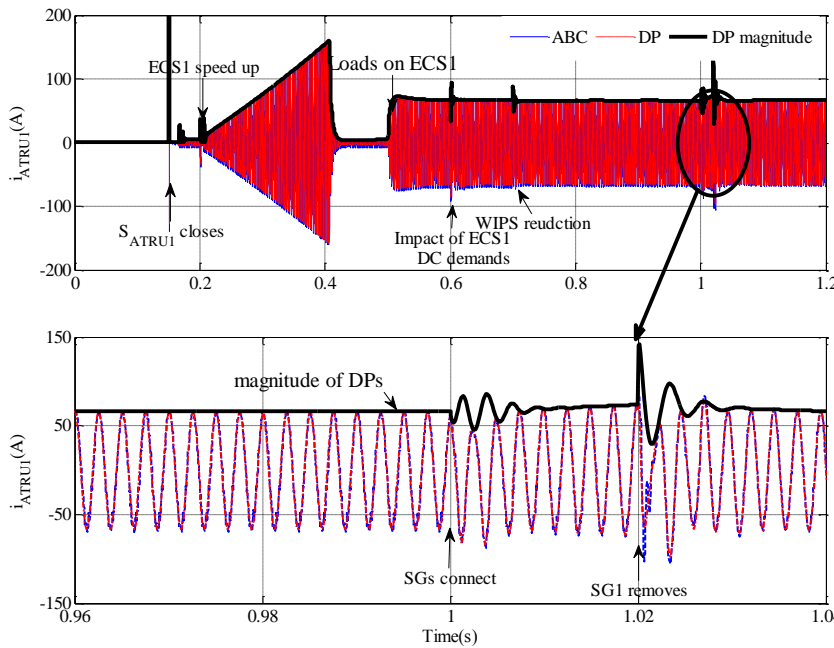

Figure 10 The dynamic response of $i_{\mathrm{HVACl}}$, phase $\mathrm{A}$ current flowing into ATRU1. Above: $\mathrm{i}_{\mathrm{HVAC}}$; below: zoom-in area of $i_{\mathrm{HVAC}}$

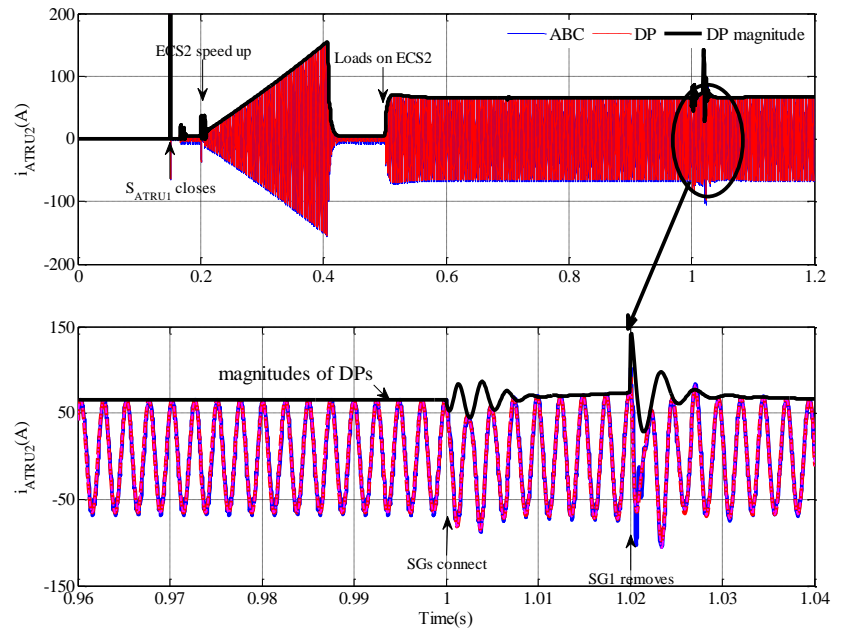

Figure 11 The dynamic response of $\mathrm{i}_{\mathrm{HVAC} 2}$, phase $\mathrm{A}$ current flowing into ATRU2 Above: $\mathrm{i}_{\mathrm{HVAC}}$; below: zoom-in area of $i_{\text {HVAC2 }}$
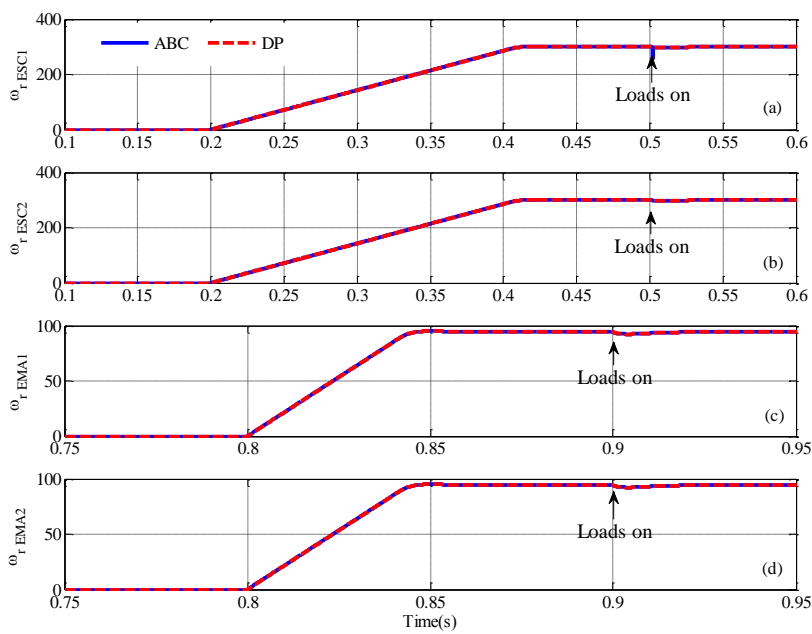

Figure 12 Dynamic response of drive loads, (a) $\omega_{\mathrm{r}_{-} \text {ECS1 }}$ speed of PMSM of ECS1; (b) $\omega_{\mathrm{r}_{-} \text {ECS2 }}$ speed of PMSM of ECS2; (c) $\omega_{\mathrm{r}_{-} \text {EMA } 1}$ speed of PMSM of

EMA1; (d) $\omega_{\text {r EMA2 }}$ speed of PMSM of EMA2

The speed of PMSMs of ECS1, ECS2, EMA1 and EMA2 is shown in Figure 12. The PMSMs are well controlled by their speed controllers. It presents well agreement between two modelling techniques.

Table 3 Comparison of the computation time between two different models

\begin{tabular}{lcc}
\hline \hline \multicolumn{1}{c}{ Model } & $A B C$ & DP \\
\hline Simulation time (s) & 7983 & 43 \\
\hline Acceleration & $\mathbf{1}$ & $\mathbf{1 8 5}$ \\
\hline
\end{tabular}


The computation time consumed by the two different models is compared in Table 3. It can be seen that the DP model is 185 times faster than the $\mathrm{ABC}$ model. The simulation time of ABC model is counted in hours compared with that of the DP model in seconds. Comparing the simulation time between the $\mathrm{ABC}$ and DP model, one can notice that the acceleration of DP model is significant. This is due to the fact that the variables are DClike in the DP model which allows larger time steps in the simulation process. Another average modelling technique based using the $d q$ frame (DQ0 model) can achieve the same acceleration order as that of DP model. However, the DQ0 model will lose its efficiency when the system is under unbalanced conditions. The DP model, on the other hand, can maintain its efficiency even the system is under unbalanced conditions. This merit of DP model has been demonstrated in our previous publications with subsystems of the aircraft EPS. Our future research will compare the three modelling techniques, ABC, DQ0 and DP models, with EPS system under MOET architecture.

\section{Conclusion}

The paper introduced a DP library for EPS studies. This library is developed in Dymola/Modelica. Based on the DP library established, a twingenerator EPS based on MOET architecture has been studied under normal conditions. The comparison between the ABC model and the DP model illustrated the accuracy of the developed DP model, during generator starting, parallel operation, load change and other events. The simulation time comparison revealed that the DP model is about 200 times faster than the ABC model. The accuracy and efficiency shows great potential of the DP modelling technique to be applied in EPS studies. In addition, since the DP modelling technique is essentially a frequency-domain modelling technique and its efficiency is not affected by the system condition, this allows the DP model a faster simulation speed even when the EPS is in unbalanced or faulty conditions. This merit of DP models will be shown in our future publications.

\section{References}

1. Emadi, K. and M. Ehsani, Aircraft power systems: technology, state of the art, and future trends. Aerospace and Electronic Systems Magazine, IEEE, 2000. 15(1): p. 28-32.

2. $\mathrm{Wu}, \mathrm{T}$. , Integrative System Modelling of Aircraft Electrical Power Systems. 2010, University of Nottingham.

3. B.Bachmann, H.W., Advanced Modeling of Electromagnetic Transients in Power Systems. Modelica Workshop 2000 Proceedings, 2000.

4. Wiesmann, H.

5. Sanders, S.R., et al., Generalized averaging method for power conversion circuits. IEEE Transactions on Power Electronics, 1991. 6(2): p. 251-259.

6. Tao, Y., S. Bozhko, and G. Asher. Assessment of dynamic phasors modelling technique for accelerated electric power system simulations. in Power Electronics and Applications (EPE 2011), Proceedings of the 2011-14th European Conference on. 2011.

7. Tao, Y., S. Bozhko, and G. Asher. Modeling of active front-end rectifiers using dynamic phasors. in Industrial Electronics (ISIE), 2012 IEEE International Symposium on. 2012.

8. Tao, Y., S. Bozhko, and G. Asher. Multigenerator system modelling based on dynamic phasor concept. in Power Electronics and Applications (EPE), 2013 15th European Conference on. 2013.

9. Yang, T., S. Bozhko, and G. Asher. Dynamic phasor modeling of autotransformer rectifier units for moreelectric aircraft. in Power Electronics and Motion Control Conference (IPEMC), 2012 7th International. 2012.

10. Yang, T., S. Bozhko, and G. Asher. Modeling of active front-end rectifiers using dynamic phasors. in Industrial Electronics (ISIE), 2012 IEEE International Symposium on. 2012.

11. Tao Yang, S.B., Greg Asher, Modeling of An 18-pulse Autotransformer Rectifier Unit with Dynamic Phasors. SAE 2012, 2012.

12. Jomier, T. More Open Electrical Technologies Techincal Report. 2009; Available from: http://www.eurtd.com/moet/. 